\title{
Behavior of the diffractive cross section in hadron-nucleus collisions
}

\author{
M. Batista, R. J. M. Covolan and A. N. Pontes \\ Instituto de Física Gleb Wataghin \\ Universidade Estadual de Campinas, Unicamp \\ 13093-970 Campinas SP Brazil
}

\begin{abstract}
A phenomenological analysis of diffractive dissociation of nuclei in proton-nucleus and mesonnucleus collisions is presented. The theoretical approach employed here is able to take into account at once data of the HELIOS and EHS/NA22 collaborations that exhibit quite different atomic mass dependences. Possible extensions of this approach to hard diffraction in nuclear processes are also discussed.
\end{abstract}

PACS numbers: 24.10.Ht, 13.85.Ni, 25.40.Ep, 25.80.Hp, 25.80.Nv, 11.55.Jy

To be published in Phys. Rev. C

\section{INTRODUCTION}

Nowadays, a lot of attention is being dedicated to experimental and theoretical studies of hadron diffractive interactions with the primary goal of investigating the Pomeron and its role in both soft and hard processes.

However, dissociation reactions in which nuclei are the diffractively excited objects are not so well known. In fact, only two experiments have dealt with this kind of process until now. The first one [1] is the analysis of the diffractive dissociation of $\mathrm{Be}, \mathrm{Al}$, and $\mathrm{W}$ in collisions with $450 \mathrm{GeV} / \mathrm{c}$ protons, performed by the HELIOS Collaboration at the CERN Super Proton Synchrotron. The second one [2] was performed at the same accelerator by the EHS/NA22 Collaboration, which has analyzed the diffractive dissociation of $\mathrm{Au}$ and $\mathrm{Al}$ excited by a 250 $\mathrm{GeV} / \mathrm{c}$ meson beam composed of $\pi^{+}$and $K^{+}$.

Probably the most striking result coming out of these experiments is that the measured target-diffraction cross sections $\sigma_{T D}$ revelead to have quite different atomic mass $(A)$ dependences. Expressing this dependence as $\sigma_{T D} \propto A^{\alpha}$, the HELIOS Collaboration has obtained $\alpha=0.35 \pm 0.02$ (that is, a value close to $1 / 3$ ), whereas the EHS/NA22 Collaboration has found $\alpha=0.58 \pm 0.06$ (not far from $2 / 3$, which corresponds to the typical $A$ dependence in nuclear inelastic interactions).

In a recent paper [3], two of us have presented an analysis of the proton inclusive spectrum obtained in protonnucleus collisions based on a multiple scattering formalism. In such an analysis, particular attention was payed to the diffractive component of the inelastic cross section $p A$ resulting in a quite good description of the experimental data of the HELIOS Collaboration. In the present paper, we extend the previous analysis to the diffractive dissociation of nuclei in meson-nucleus collisions.

\section{THEORETICAL FRAMEWORK}

Our starting point is the discussion of the diffractive dissociation cross section for hadron-proton $(h p)$ colli- sions (in this case, $h=p, \pi^{+}, K^{+}$). In the diffractive region, the invariant cross section of an inclusive process of the type $h p \rightarrow h^{\prime} X$ is given by

$$
E \frac{d^{3} \sigma}{d \mathbf{p}^{3}}=\frac{s}{\pi} \frac{d^{2} \sigma}{d t d M_{X}^{2}}
$$

with

$$
-t=m_{h^{\prime}}^{2}(1-x)^{2} / x+p_{T}^{2} / x,
$$

where $x=2 p_{L} / \sqrt{s}$ is the Feynman variable of the particle $h^{\prime}$ and $M_{X}^{2}$ is the invariant mass diffractively excited, which is defined as $M_{X}^{2} \equiv\left(p_{h}+p_{p}-p_{h^{\prime}}\right)^{2}$. Usually variable $\xi=M_{X}^{2} / s=1-x$ is also employed to describe this sort of process.

In the approach used here, the single diffractive cross section, Eq. (1), is expressed in terms of a modified version of the Triple Pomeron model [4] which was adequate to prevent against unitarity violation (see [5]):

$$
\frac{d^{2} \sigma_{S D}}{d \xi d t}(h p \rightarrow h X)=f_{R}^{h}(\xi, t) \times \sigma_{\mathbb{P}_{p}}(s \xi)
$$

where $f_{R}^{h}$ is the renormalized Pomeron flux factor [5], that is

$$
f_{R}^{h}(\xi, t)=\frac{f_{S}^{h}(\xi, t)}{N(s)}
$$

with the standard flux factor given by the DonnachieLandshoff expression [6]

$$
f_{S}^{h}(\xi, t)=\frac{\beta_{h}^{2}}{16 \pi} F^{2}(t) \xi^{[1-2 \alpha} \mathbb{P}^{(t)]}
$$

and

$$
N(s)=\int_{1.5 / s}^{1} \int_{-\infty}^{0} f_{S}^{h}(\xi, t) d t d \xi .
$$

In Eq. (5), $\beta_{h}$ stands for the hadron-pomeron couplings at the quasi-elastic vertices, whose values can be obtained directly from the analysis of the total cross sections reported in Ref. [7]. With a suitable change of normalization these values result to be $\beta_{p}=6.82 \mathrm{GeV}^{-1}$, 
$\beta_{\pi}=4.13 \mathrm{GeV}^{-1}$ and $\beta_{K}=3.71 \mathrm{GeV}^{-1}$. In the same equation, $F(t)$ corresponds to the hadron form factor. When a proton is the recoiling particle, $F(t)$ is given by the Dirac form factor,

$$
F_{1}(t)=\frac{\left(4 m_{p}^{2}-2.79 t\right)}{\left(4 m_{p}^{2}-t\right)} \frac{1}{\left(1-\frac{t}{0.71}\right)^{2}}
$$

for pions and kaons, we apply the dipole formula

$$
F_{1}(t)=\frac{1}{\left(1-\frac{t}{\mu_{m}^{2}}\right)^{2}}
$$

with $\mu_{\pi}^{2}=0.92 \mathrm{GeV}^{2}$ and $\mu_{K}^{2}=1.10 \mathrm{GeV}^{2}$.

In Eq. (3), the pomeron-proton cross section is written as

$$
\sigma_{\mathbb{P}_{p}}\left(M_{X}^{2}\right)=\beta_{p} g_{\mathbb{P}}(s \xi)^{\epsilon}
$$

where $g_{\mathbb{I P}}=0.87 \mathrm{GeV}^{-1}$. The pomeron trajectory is always $\alpha(t)=1+\epsilon+\alpha^{\prime} t$, with $\epsilon=0.104$ and $\alpha^{\prime}=0.25$ $\mathrm{GeV}^{-2}$ obtained in [7]. Eqs. (1)-(9) completly specify how one calculates the diffractive cross section for the processes $p p \rightarrow p X, \pi^{+} p \rightarrow \pi^{+} X$ and $K^{+} p \rightarrow K^{+} X$.

\section{NUCLEAR DIFFRACTIVE DISSOCIATION}

Now, we turn our attention to diffraction in nuclear collisions. As proposed in [8], the invariant cross section for the inclusive reaction $h A \rightarrow h X$ is expressed in terms of

$$
\frac{d^{3} \sigma}{d x d p_{T}^{2}}(h A \rightarrow h X)=\sum_{\nu=1}^{A} \sigma_{\nu}^{h A} D_{\nu}^{N}\left(x, p_{T}^{2}\right),
$$

where $\sigma_{\nu}^{h A}$ is the partial inelastic cross section resulting of $\nu$ interactions:

$$
\sigma_{\nu}^{h A}=\int d^{2} b \frac{A !}{\nu !(A-\nu) !} P_{A}^{\nu}(b)\left[1-P_{A}(b)\right]^{A-\nu} .
$$

$P_{A}(b)$ is the probability of a nucleon to suffer an inelastic interaction at a given impact parameter $b$, which is expressed in terms of the nuclear density through the relationship

$$
P_{A}(b)=\sigma_{\text {inel }}^{h p} \int_{-\infty}^{+\infty} d z \rho_{A}(z, b) .
$$

The nuclear densities applied here are the same as those used in 3] and respect the normalization condition $\int d^{3} r \rho_{A}(r)=1$ For light nuclei $(6 \leq A \leq 18)$ they follow the expression 1

\footnotetext{
${ }^{1}$ We notice that a factor $1 / A$ is missing in the parametrization corresponding to Eq. (13) in the Appendix of Ref. [3].
}

$\rho(r)=\frac{4}{\pi^{3 / 2} a_{0}^{3} A}\left[1+\frac{1}{6}(A-4) \frac{r^{2}}{a_{0}^{2}}\right] \exp \left(-r^{2} / a_{0}^{2}\right)$,

with $a_{0}=\left[\left(r_{0}^{2}-r_{p}^{2}\right)^{1 / 2} /(5 / 2-4 / A)\right]^{1 / 2}, r_{0}=1.2 A^{1 / 3}$ $\mathrm{fm}$, and $r_{p}=0.8 \mathrm{fm}$. For heavier nuclei $(A \geq 18), \rho(r)$ is calculated according to the Woods-Saxon formula [9], that is

$$
\rho(r)=\frac{c_{0}}{1+\exp \left[\left(r-r_{0}\right) / b_{0}\right]},
$$

where $c_{0}$ is the normalization constant

$$
c_{0}=\frac{3}{4 \pi r_{0}^{3}} \frac{1}{1+\left(b_{0} \pi / r_{0}\right)^{2}}
$$

and $b_{0}=0.4 \mathrm{fm}$.

In order to calculate the inelastic cross sections $\sigma_{\text {inel }}^{h p}$ appearing in Eq. (12), we introduce the following parametrizations (already in milibarns) which are based on Regge phenomenology:

$$
\begin{gathered}
\sigma_{\text {inel }}^{p p}=12.37 s^{0.104}+34.90 s^{-0.20}-31.30 s^{-0.54}, \\
\sigma_{\text {inel }}^{\pi p}=7.86 s^{0.104}+20.49 s^{-0.20}-8.02 s^{-0.54}, \\
\sigma_{\text {inel }}^{K p}=6.46 s^{0.104}+20.46 s^{-0.20}-27.3 s^{-0.54} .
\end{gathered}
$$

The distribution $D_{\nu}^{N}\left(x, p_{T}^{2}\right)$ is generated by a recurrence formula that starts with the assumption (see [3] for details)

$$
D_{\nu=1}^{N}\left(x, p_{T}^{2}\right)=\frac{1}{\sigma_{\text {inel }}^{h p}}\left(\frac{d^{3} \sigma}{d x d p_{T}^{2}}\right)^{h N \rightarrow p X} .
$$

To obtain the total inclusive cross section for the process $h A \rightarrow h X$, the sum over $\nu$ in Eq. (10), which represents the number of times that the incident particle is scattered in the nuclear environment, should in principle run until its maximum valuet.

However, when only diffractive events are concerned, it is enough to put $\nu=1$ since these processes are supposed to take place through single peripheral interactions with the outlying nucleons. Based on this argument, we use Eqs. (10) and (19) to express the single diffractive component of the invariant cross section in hadron-nucleus collisions as

$$
\frac{d^{2} \sigma_{S D}}{d \xi d t}(h A \rightarrow h X)=\frac{\sigma_{\nu=1}^{h A}}{\sigma_{\text {inel }}^{h p}} \frac{d^{2} \sigma_{S D}}{d \xi d t}(h p \rightarrow h X),
$$

which can be entirely calculated from what has been established previously. Integrating Eq. (20) over $\xi$ and $t$, one obtains for the single diffractive hadron-nucleus cross section a very simple relationship:

$$
\sigma_{S D}^{h A \rightarrow h X}=\frac{\sigma_{\nu=1}^{h A}}{\sigma_{\text {inel }}^{h p}} \sigma_{S D}^{h p \rightarrow h X} .
$$

\footnotetext{
${ }^{2}$ In practice, not all terms need to be calculatedin order to obtain a quite good description of the data (see [3]).
} 


\section{RESULTS AND DISCUSSION}

A comparison of the model outlined above with cross section data has already been presented in Ref. [3] for the case of $p A \rightarrow p X$ with a slightly different parametrization. Here we show in Fig. 1 the differential cross section as a function of the invariant mass $M_{X}^{2}$ compared to the EHS/NA22 data [2] which are given in terms of the normalized distribution $1 / N_{e v}\left(d N_{e v} / d M_{X}^{2}\right)$ for the reactions $\left(\pi^{+} / K^{+}\right) A l \rightarrow\left(\pi^{+} / K^{+}\right) X$ and $\left(\pi^{+} / K^{+}\right) A u \rightarrow$ $\left(\pi^{+} / K^{+}\right) X$ (in these data there is no distinction between pions and kaons).

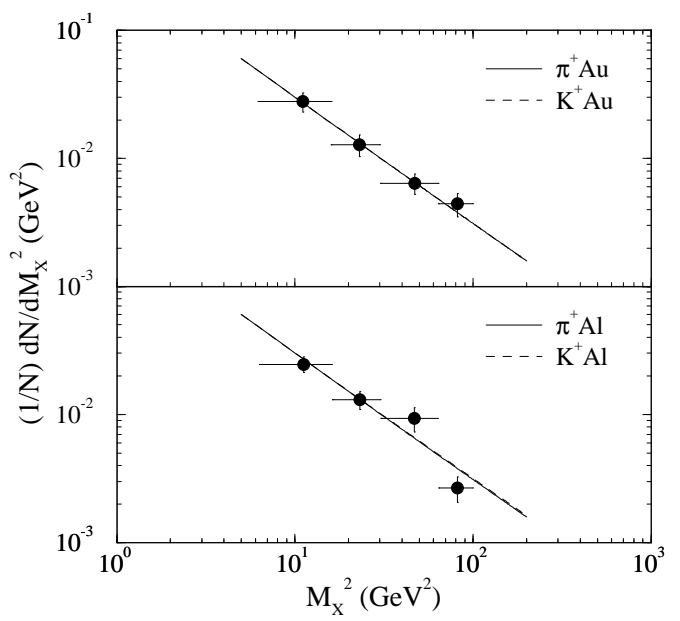

FIG. 1. Normalized $M_{X}^{2}$ distributions. The experimental data are from [2]

In Fig. 2, theoretical predictions for the cross section $\sigma_{S D}^{h A \rightarrow h X}$, given by Eq. (21) with $h=p, \pi^{+}, K^{+}$, are compared with data of both collaborations, HELIOS [1] and EHS/NA22 [2]. As can be seen, the model provides a quite good description for the $A$ dependence of the cross section for the various reactions.

This last result can be better understood if we construct a toy model by assuming for the nuclear densities the (over)simplified form,

$$
\rho(r)=\frac{1}{\pi^{3 / 2} d_{0}^{3}} e^{-r^{2} / d_{0}^{2}} .
$$

In such a case, all calculations can be performed analitically and the nuclear diffractive cross section results to be

$$
\sigma_{S D}^{h A \rightarrow h X}=\pi d_{0}^{2}\left[1-\left(1-\frac{\sigma_{i n e l}^{h p}}{\pi d_{0}^{2}}\right)^{A}\right] \frac{\sigma_{S D}^{h p \rightarrow h X}}{\sigma_{\text {inel }}^{h p}} .
$$

Although such a formula is not appropriate for quantitative analysis, it is quite interesting from a qualitative point of view because it gives us a hint of how the "elementary" inelastic cross section distinguishes the $A$ dependence of the different reactions: the higher $\sigma_{\text {inel }}^{h p}$ is, the flatter the $A$ dependence of the term within square brackets becomes. Based on this argument, the behavior observed in Fig. 2 is easily understood since $\sigma_{\text {inel }}^{p p}>\sigma_{\text {inel }}^{\pi^{+} p}>\sigma_{\text {inel }}^{K^{+} p}$. Of course, the real calculation is not as simple as Eq. (23), however the influence of $\sigma_{\text {inel }}^{h p}$ in the $A$ dependence of the term $\sigma_{\nu=1}^{h A}$ in Eq. (21) is quite the same.

As we have seen, the phenomenological model presented here provides a satisfactory description for soft nuclear diffraction. Now, let us make some conjectures about how it might employed to make predictions on hard diffractive processes generated by nuclear collisions. Before going to the point, let us make a brief digression.

The possibility for diffractive interactions occur in hard regime was first proposed by the Ingelman-Schlein (IS) model [10]. According to this model, a diffractive reaction may take place as a two-step process in which (1) a Pomeron is emitted from the quasi-elastic vertex and then (2) partons of the Pomeron interact with partons of the other hadron giving rise to dijets production or any other hard process. In fact, the very concept of Pomeron flux factor, which appears in Eq. (3), was introduced to represent the first step of such a process, being defined as

$$
f(\xi, t) \equiv \frac{1}{\sigma_{\mathbb{P}_{p}}(s \xi)} \frac{d^{2} \sigma_{S D}}{d \xi d t}
$$

The second step is calculated through the QCD parton model and requires the knowledge of the Pomeron structure function.

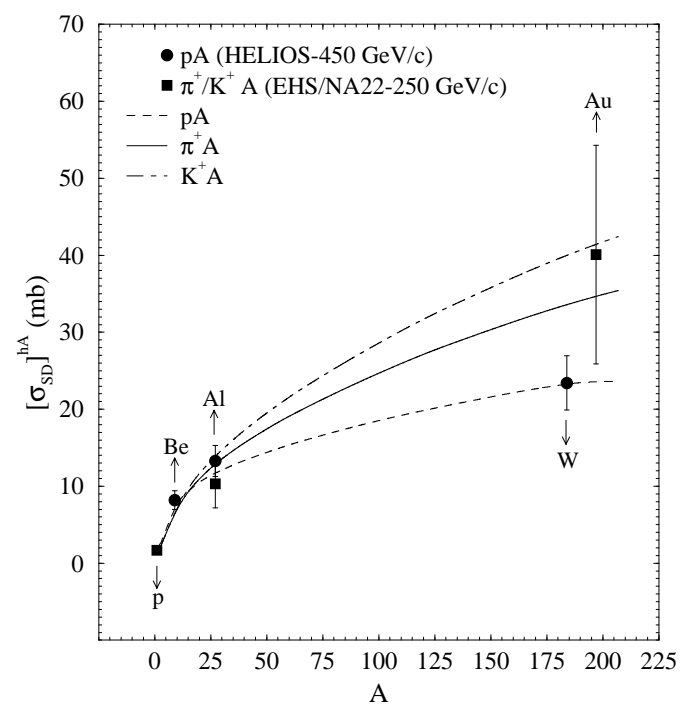

FIG. 2. Total cross section for nuclear diffractive dissociation. The elements corresponding to the nuclear targets are indicated by the arrows. The data are from [1] and [2]

Discussion on these themes can be found, for instance, in two recent works which reported a study on the Pomeron structure function [11] and an extensive analysis of the role of the standard and renormalized Pomeron flux factors in the diffractive production of $W$ 's and dijets at the DESY HERA and Fermilab Tevatron colliders [12]. This analysis 12] has shown that the standard flux factor 
is ruled out by the data and that the renormalized flux, which presents good results for diffractive hadroproduction, has serious difficulties in diffractive photoproduction. In order to overcome such difficulties, the IS model was reproposed in 12] in a modified form such that the Pomeron flux factor is replaced by a new distribution called diffraction factor which is defined as

$$
F_{S D}(\xi, t) \equiv \frac{1}{\sigma_{S D}^{h p}} \frac{d^{2} \sigma_{S D}^{h p}}{d \xi d t}
$$

This new approach presents good results for both hadroproduction and photoproduction of dijets.

Turning to nuclear diffraction, we see that replacing Eq.(3) into Eq.(20) one gets

$$
\frac{d^{2} \sigma_{S D}}{d \xi d t}(h A \rightarrow h X)=f(\xi, t) \times \frac{\sigma_{\nu=1}^{h A}}{\sigma_{\text {inel }}^{h p}} \sigma_{\mathbb{P}_{p}}(s \xi),
$$

where the flux factor $f(\xi, t)$ was left free to assume whichever form (standard or renormalized).

Now, considering how to apply the previous ideas to estimate the rates of some particular hard nuclear diffractive process (supposed to occur), one might think about two possibilities:

1) Sticking to the original IS model (namely, normalization of the differential cross section by $\sigma_{\mathbb{P}_{p}}$ ), one could define from Eq.(26) a new flux factor that would depend on $A$,

$$
f_{A}(\xi, t) \equiv \frac{1}{\sigma_{\mathbb{P}_{p}}(s \xi)} \frac{d^{2} \sigma_{S D}}{d \xi d t}(h A \rightarrow h X)=f(\xi, t) \frac{\sigma_{\nu=1}^{h A}}{\sigma_{\text {inel }}^{h p}} ;
$$

2) On the other hand, it seems natural to define a sort of Pomeron-nucleus cross section by

$$
\sigma_{\mathbb{P}_{A}}(s \xi) \equiv \frac{\sigma_{\nu=1}^{h A}}{\sigma_{\text {inel }}^{h p}} \sigma_{\mathbb{P}_{p}}(s \xi)
$$

and consider the flux factor defined by,

$$
f(\xi, t) \equiv \frac{1}{\sigma_{\mathbb{P} A}(s \xi)} \frac{d^{2} \sigma_{S D}}{d \xi d t}(h A \rightarrow h X),
$$

as the correct quantity to apply in the calculations. Of course, these definitions would imply in quite different predictions.

Alternatively one could consider, instead of the flux factor, the idea of diffraction factor, Eq.(25). Since the integral of this distribution is normalized to the unity by definition [12], we see that its application to the hadronnucleus case results to be the same as for the hadronproton case, that is

$$
F_{S D}(\xi, t)=\frac{1}{\sigma_{S D}^{h A}} \frac{d^{2} \sigma_{S D}^{h A}}{d \xi d t}=\frac{1}{\sigma_{S D}^{h p}} \frac{d^{2} \sigma_{S D}^{h p}}{d \xi d t}
$$

once Eqs. (20) and (21) are taken into account. Therefore, with the concept of diffraction factor, there is no ambiguity about which expression to use.

It is important to notice however that, whatever be the choice, Eq. (27), (29), or (30), the previous discussion establish the main elements needed to perform the calculations and make predictions of hard diffraction in nuclear collisions in the same spirit that has been done in 12 for proton-antiproton interactions. The only new input necessary are parametrizations of structure functions obtained for nuclear environments. In this sense what is being proposed above is a sort of extension of the Ingelman-Schlein model (in its original and modified forms) to take into account hard diffraction in nuclear processes.

In summary, we have presented in this paper a phenomenological approach that provides a quite good description of the apparently discrepant $A$ dependence observed in the diffractive cross sections of meson-nucleus and proton-nucleus collisions. The model used for such allows one to imagine possible theoretical frameworks within which one could make predictions on hard diffractive processes originated from hadron-nucleus interactions.

\section{ACKNOWLEDGMENTS}

We would like to thank the Brazilian governmental agencies CNPq, CAPES and FAPESP for financial support.

[1] HELIOS Collab., T. Åkesson et al., Z. Phys. C 49, 355 (1991).

[2] EHS/NA22 Collab., N. M. Agababyan et al., Z. Phys. C 72, 65 (1996).

[3] M. Batista and R. J. M. Covolan, Phys. Rev. C 60, 014902 (1999).

[4] P. D. B. Collins, An Introduction to Regge theory and high energy physics (Cambridge University Press, Cambridge, England, 1977); P. D. B. Collins and A. D. Martin, Hadron Interactions (Adam Hilger Ltd., Bristol, England, 1984).

[5] K. Goulianos, Phys. Lett. B 358, 379 (1995); B 363, 268 (E) (1995); K. Goulianos and J. Montanha, Phys. Rev. D 59, 114017 (1999).

[6] A. Donnachie and P. V. Landshoff, Nucl. Phys. B 303, 634 (1988).

[7] R. J. M. Covolan, J. Montanha, K. Goulianos, Phys. Lett. B 389, 176 (1996).

[8] G. M. Frichter, T. K. Gaisser and T. Stanev, Phys. Rev. D 56, 3135 (1997).

[9] R. C. Barret and D. F. Jackson, Nuclear Sizes and Structure (Oxford University Press, Oxford, 1977). 
[10] G. Ingelman and P. E. Schlein, Phys. Lett. B 152, 256 (1985).

[11] R. J. M. Covolan and M. S. Soares, Phys. Rev. D 57, 180 (1998).

[12] R. J. M. Covolan and M. S. Soares, Phys. Rev. D 60, 054005 (1999); Erratum: Phys. Rev. D 61, 19901 (2000). 